\title{
A Handover Management Scheme for Mobile IPv6 Networks
}

\author{
A.K.M. Mahtab Hossain and Kanchana Kanchanasut \\ Internet Education and Research Laboratory \\ Asian Institute of Technology \\ P.O.Box 4, Klong Luang, Pathumthani 12120, Thailand \\ Email: <mahtab@interlab.ait.ac.th,kk@cs.ait.ac.th>
}

\begin{abstract}
Mobile IPv6 provides IP layer mobility management for IPv6 networks. Despite many advantages it offers in comparison to Mobile IPv4, handover management still remains an issue for Mobile IPv6. For real-time connections, the handover latency and signaling overhead incurred by the protocol may become significant. This paper presents a new method named Crossover MAP based Hierarchical Mobile IPv6 (XMAP-HMIPv6) which inherits the advantage of Hierarchical Mobile IPv6 of reducing signaling load for inter-domain mobility. We incorporate an analytical model for the performance analysis of this new scheme along with other existing handover management proposals. Finally, we present analytical results in different environments and compare our scheme with other existing proposals. The analytical model results show that, our scheme performs almost similar to $[\mathrm{F}+\mathrm{H}] \mathrm{MIPv6}$ in terms of reducing handover latency and outperforms both HMIPv6 and MIPv6.
\end{abstract}

\section{INTRODUCTION}

IP layer mobility provides seamless connectivity to the Internet. Mobility in IPv4 [6] is an after-thought whereas IPv6 [8] is designed with mobility in mind. As a result, Mobile IPv6 [2] has evolved remarkably compared to Mobile IPv4 [1]. Mobile IPv6 is developed to facilitate the roaming of mobile devices in different wired or wireless networks while maintaining a permanent IP address.

Each time a mobile node (MN) moves to a new link, its IP address needs to be changed for the inherent characteristics of IP routing. Therefore, the packets destined to its previous IP address will not be able to reach the MN. In Mobile IPv6 (MIPv6), each MN is always identified by its home address (HoA), regardless of its current point of attachment to the Internet. IPv6 packets addressed to an MN's HoA are transparently routed to its current point of attachment by home agent (HA) of the MN, situated in its home network. MIPv6 also enables correspondent hosts $(\mathrm{CHs})$ to cache the binding of its HoA with its care-of address (CoA) so that they can send any packets directly to the MN at its CoA.

However, as the MN roams from one network to another, even within the same domain, Mobile IPv6's intrinsic design requires it to update the $\mathrm{HA}$ and all the $\mathrm{CHs}$ of its new point of attachment. These registration procedures cause delay for the packets and also require additional signaling. Delay sensitive applications like real-time communications perform poorly for that matter. So, many extensions to the MIPv6 have been proposed which assist the $\mathrm{MN}$ to acquire smooth handover. We discuss here those most relevant to our own work.

Fast Mobile IPv6 (FMIPv6) [7] enables the MN to achieve a potential $\mathrm{CoA}$ at the new subnet before the $\mathrm{MN}$ actually attaches to it. A temporary tunnel between the previous access router (PAR) and the new access router (NAR) is set up before the MN attaches to the new subnet. So, packets destined for the MN are already forwarded to its new CoA. Once attached to the new subnet, The MN only needs to announce its presence to receive those buffered packets.

Hierarchical Mobile IPv6 (HMIPv6) [3] relaxes the requirement of MIPv6 that the MN should inform its current IP address to its HA and to all the CHs every time its point of attachment changes. In HMIPv6 protocol, every domain has mobility management agents named mobility anchor points (MAPs) which serves as a local HA for the MN and the $\mathrm{MN}$ will maintain two IP addresses - one is the on-link CoA (LCoA) and the other is the regional CoA (RCoA). Whenever the $\mathrm{MN}$ enters into a domain, it first registers with an MAP entity. In the process, it acquires a RCoA on that MAP's link. After that, the MN uses this address to register with its HA and all the CHs. As long as the MN moves only inside that MAP's domain, the movement remains transparent to the nodes (including its HA) outside the domain. Only the LCoA changes which the MN informs only to the MAP entity. IPv6 packets destined to the MN reaches the MAP first and consequently, the MAP tunnels the packet to the MN's LCoA.

The combination of Fast and Hierarchical Mobile IPv6 $([\mathrm{F}+\mathrm{H}] \mathrm{MIPv} 6)[3]$ tries to incorporate the advantages of FMIPv6 and HMIPv6 together. Like FMIPv6, it aims to reduce the handover latency, thereby, minimizing the delay and packet losses. On the other hand, it tries to use bandwidth efficiently by incurring less signaling overhead for each movement of the $\mathrm{MN}$ inside an administrative domain, similar to HMIPv6.

In flow-based fast handover for Mobile IPv6 [5] [4], 
when the MN attaches to a new subnet, it probes for the cross-over router - the first router common to the path from the HA to its old CoA and to the new CoA. This protocol has the requirement that, every router should maintain flow information (i.e., source, destination and flow label) of each traffic flow it sends, receives or routes. Hop by hop extension header having old flow information is added to the MIPv6 binding update (BU) message which is processed at every router on its path to the HA. The first router in the path which has the flow information included in the hop by hop frame is the desired cross-over router. From this router, the traffic destined for the $\mathrm{MN}$ is now diverted from MN's old CoA towards the MN's new CoA. Actually, a temporary tunnel is created from the cross-over router to MN's new CoA and this tunnel is active for a time period sufficient enough to let the $\mathrm{MN}$ complete its registration with its $\mathrm{HA}$ and all the $\mathrm{CHs}$.

In this paper, we present a new method named Cross-over MAP based Hierarchical Mobile IPv6 (XMAP-HMIPv6). This method causes reduced signaling load by separating micro mobility (movement inside an administrative domain) from macro mobility (movement across domains) like HMIPv6. But unlike HMIPv6, it always finds the cross-over MAP (the first MAP, common to the path from the HA to its old LCoA, and from the HA to its new LCoA) to divert the traffic for the $\mathrm{MN}$ and results in reduced handover latency. The proposed method requires a few modifications on MAP functionality and also slight change in BU and binding acknowledgement (BAck) message formats and their processing.

The remainder of the paper is organized as follows: Section II describes the proposed XMAP-HMIPv6 method. The analytical analytical mobility model is described in section III. Section IV derives the cost functions using the analytical model. The results are presented in section V. Finally, in section VI, we discuss conclusions and future work.

\section{XMAP-HMIPv6 METHOD}

This section presents a new method named "Cross-over MAP based Hierarchical Mobile IPv6 (XMAP-HMIPv6)" for host mobility management. The XMAP-HMIPv6 method incurs less signaling overhead by separating micro mobility from macro mobility just like HMIPv6. But, unlike HMIPv6, where the MAP selection criteria is MN-dependent and does not have strict containment, XMAP-HMIPv6 always finds the cross-over MAP to divert the traffic destined for the $\mathrm{MN}$ in case of micro mobility. For macro mobility, it performs similar to standard MIPv6 protocol. XMAP-HMIPv6 introduces new functions to the MAP entity and minor extensions to $\mathrm{MN}$ and HA operation in case of BU and BAck processing. The correspondent host operation remains the same.

Whenever the MN attaches to a new point of attachment, it configures LCoA (on-link CoA) with stateless autoconfiguration [9]. In Fig. 1, at first, the $\mathrm{MN}$ enters into a new administrative domain (Macro-Move), after which, it sends a
BU (with hop by hop extension header carrying its link-layer address, home address and LCoA) towards the HA.
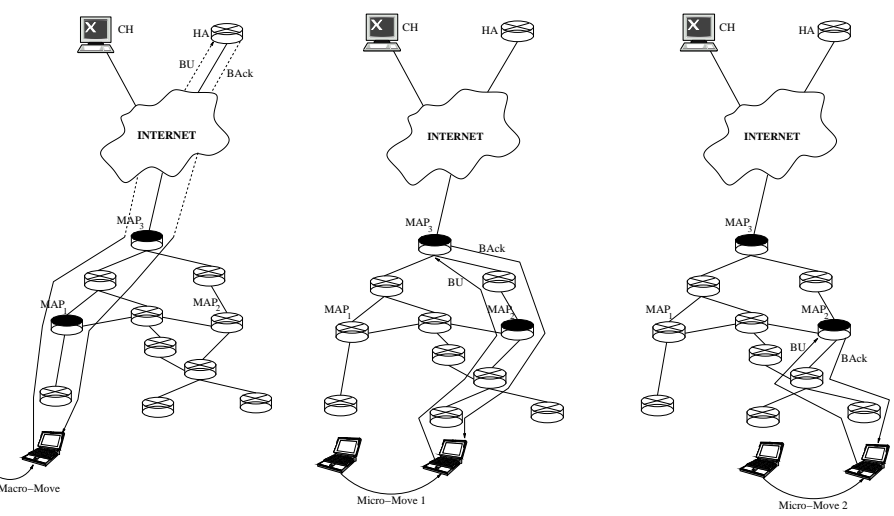

Fig. 1. Three consecutive movements of the MN. At first, the MN enters into a domain (Macro-Move) and then, it moves twice inside the domain (Micro-Move 1 \& Micro-Move 2)

Every router along the path will parse the hop by hop extension header but XMAP-HMIPv6 options within the header cause only the MAPs (not the other routers in the administrative domain) to process this header. After receiving the BU, an MAP first checks to see whether it has an entry for the concerned $\mathrm{MN}$ in the binding cache already (using the home address option provided inside the header). If it does not, it generates a regional care-of address (RCoA) for the MN using the LLA option of the MN provided inside the header and its own subnet-prefix. The MAP then creates a new binding cache entry for the MN, binding the created RCoA and alternate $\mathrm{CoA}$ of the $\mathrm{MN}$ provided inside the header. After that, the MAP creates a new similar hop by hop extension header by putting the generated RCoA in the alternate care-of address field and copying all the other fields of the received BU's hop by hop extension header. The MAP then encapsulates the original BU and adds the new hop by hop extension header to it and sends it towards HA.

All the MAPs along the path perform similar operations on the received BU until it reaches the Gateway MAP. After performing the similar operations as a usual MAP, the Gateway MAP sets or clears some bits (e.g. $D=1, M=0 \& X=1$ ) in XMAP-HMIPv6 options inside the hop by hop extension header, ensuring that, the other domain MAPs do not process this header. Here, $\mathrm{D}=1$ means only the destination should process this extension header, $M=1$ means the MAPs should process this header and $\mathrm{X}=1$ means the administrative domain the MN has stepped in, supports XMAP-HMIPv6.

Finally, the BU reaches the HA which decapsulates the BU to retrieve the original $\mathrm{BU}$ sent by the $\mathrm{MN}$. In the process, the HA collects all the regional care-of addresses of the MN (addresses of the MN at MAPs' subnets) and all the MAPs' addresses from the BU (from the source address field). Then, the HA sends BAck with "Type 2 Routing Header" including all the MAPs' addresses in-order so that it travels through all the MAPs that encapsulated the original BU. In this way, all the entries in their binding cache are acknowledged. The HA 
also informs the $\mathrm{MN}$ about all of its regional care-of addresses by putting them inside the mobility options header. While sending binding updates to $\mathrm{CH}$, the MN follows HMIPv6 protocol providing only the RCoA at its Gateway MAP's link in the alternate CoA field.

TABLE I

Binding CACHE ENTRIES AFTER MACRO-MOve

\begin{tabular}{|c|c|c|c|c|}
\hline Router & \multicolumn{4}{|c|}{ Binding Cache } \\
\hline & $\begin{array}{c}\text { Home } \\
\text { Address }\end{array}$ & $\begin{array}{c}\text { Regional } \\
\text { CoA }\end{array}$ & $\begin{array}{c}\text { Care-of } \\
\text { Address }\end{array}$ & Lifetime \\
\hline $\mathrm{HA}^{*}$ & HoA & & $\mathrm{RCoA}_{3}$ & \\
\hline $\mathrm{MAP}_{3}$ & HoA & $\mathrm{RCoA}_{3}$ & $\mathrm{RCoA}_{1}$ & \\
\hline $\mathrm{MAP}_{1}$ & $\mathrm{HoA}$ & $\mathrm{RCoA}_{1}$ & $\mathrm{LCoA}_{1}$ & \\
\hline
\end{tabular}

TABLE II

Binding CACHe ENTRIES AFTer Micro-Move 1

\begin{tabular}{|c|c|c|c|c|}
\hline Router & \multicolumn{4}{|c|}{ Binding Cache } \\
\hline & $\begin{array}{c}\text { Home } \\
\text { Address }\end{array}$ & $\begin{array}{c}\text { Regional } \\
\text { CoA }\end{array}$ & $\begin{array}{c}\text { Care-of } \\
\text { Address }\end{array}$ & Lifetime \\
\hline HA & HoA & & $\mathrm{RCoA}_{3}$ & \\
\hline $\mathrm{MAP}_{3}^{*}$ & $\mathrm{HoA}$ & $\mathrm{RCoA}_{3}$ & $\mathrm{RCoA}_{2}$ & \\
\hline $\mathrm{MAP}_{2}$ & $\mathrm{HoA}$ & $\mathrm{RCoA}_{2}$ & $\mathrm{LCoA}_{2}$ & \\
\hline
\end{tabular}

TABLE III

Binding CACHe ENTRIES AFter Micro-Move 2

\begin{tabular}{|c|c|c|c|c|}
\hline Router & \multicolumn{4}{|c|}{ Binding Cache } \\
\hline & $\begin{array}{c}\text { Home } \\
\text { Address }\end{array}$ & $\begin{array}{c}\text { Regional } \\
\text { CoA }\end{array}$ & $\begin{array}{c}\text { Care-of } \\
\text { Address }\end{array}$ & Lifetime \\
\hline HA & HoA & & $\mathrm{RCoA}_{3}$ & \\
\hline $\mathrm{MAP}_{3}$ & HoA & $\mathrm{RCoA}_{3}$ & $\mathrm{RCoA}_{2}$ & \\
\hline $\mathrm{MAP}_{2}^{*}$ & $\mathrm{HoA}$ & $\mathrm{RCoA}_{2}$ & $\mathrm{LCoA}_{3}$ & \\
\hline
\end{tabular}

On the contrary, when the $\mathrm{MN}$ moves locally inside an administrative domain, as shown in Fig. 1 (Micro-Move 1 $\& 2$ ), it sends the BU in the same way. But this time, the cross-over MAP intercepting the BU, finds an entry matching the home address of the MN. So, it sends the BAck in a similar way as HA, causing it to travel through all the lowerlevel MAPs that encapsulated the BU and also providing all the RCoAs of the MN at those MAP's links (including the $\mathrm{RCoA}$ at its own link). The $\mathrm{MN}$ receiving the BU, updates its RCoAs entries, starting from the cross-over MAP link's $\mathrm{RCoA}$ to the lowest-level addresses (i.e. LCoA) with the new addresses provided inside the BAck.

Consider the MN's three consecutive movements as shown in Fig. 1. After the completion of each movement, the binding cache entries for the HA and concerned MAPs are shown in in Table I, II and III. The cross-over MAP where the binding process ends, is also marked with asterisk in the tables. For macro movement, no cross-over MAP is found, so, the BU message should travel to HA. We also notice from the tables that, above the cross-over router, the bindings remain intact and there is an additional entry named "Regional CoA" for the MAP's binding cache which is needed for hierarchical tunneling of the packets.

Suppose, after the macro and two micro movements of the

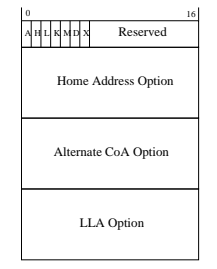

Fig. 2. XMAP-HMIPv6 Options inside the hop by hop extension header

$\mathrm{MN}$ as indicated in Fig. 1, a $\mathrm{CH}$ wants to send a packet to the MN. If the MN has already updated its bindings with that $\mathrm{CH}$, then it addresses the packet to $\mathrm{RCoA}_{3}$. Otherwise, the $\mathrm{CH}$ addresses the packet to the MN's home address which is eventually forwarded by the MN's HA to the Gateway MAP. So, in either case, the packet reaches the Gateway MAP $\left(\mathrm{MAP}_{3}\right.$ in Fig. 1). The Gateway MAP then looks up its binding cache (i.e. $\mathrm{MAP}_{3}$ entry in Table III) to extract the $\mathrm{CoA}$ of the $\mathrm{MN}$ (i.e. $\mathrm{RCoA}_{2}$ ). Then it encapsulates the original packet and addresses the new packet to $\mathrm{RCoA}_{2}$. So, this packet is intercepted by $\mathrm{MAP}_{2}$ in the same way as $\mathrm{MAP}_{3}$, which consults its binding cache to find the $\mathrm{CoA}$ (i.e. $\mathrm{LCoA}_{3}$ ) of the $\mathrm{MN}$ in a similar manner. So, the packets for the MN from either HA or $\mathrm{CH}$ travels through all the registered MAPs which keeps tunneling it to the lower-level regional care-of address until it reaches the lowest level MAP. The lowest-level MAP tunnels the packet to the current CoA of the MN. The MN send packets normally to the $\mathrm{HA}$ or $\mathrm{CH}$ with $\mathrm{RCoA}_{3}$ as the source address or it may reverse tunnel the packets through the Gateway MAP if it needs to use $\mathrm{LCoA}_{3}$ as the source address in the packet.

\section{AnAlyticAl Mobility Model}

\section{A. Network Description}

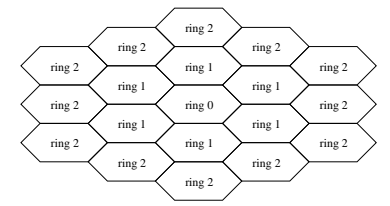

Fig. 3. Ring Structure of the Access Routers

In this section, we incorporate an analytical mobility model [10] to evaluate the performance of our XMAP-HMIPv6 proposal against other protocols such as MIPv6, HMIPv6 and $[\mathrm{F}+\mathrm{H}] \mathrm{MIPv} 6$. We consider layer-3 handover delay as our performance metric. We assume the wireless IP network to have hexagonal cell structure and the proposed analytical model to be based on random-walk mobility model [11]. We assume that each hexagonal cell area is served by a distinct 
access router. So, the access routers can be thought of arranged in ring structures as shown in Fig. 3. A ring $k$ is composed of $6 k$ cells or access routers expect for ring 0 which has only one cell. Suppose the administrative domain is composed of $R$ such rings. So the total number of cells comprising the domain,

$$
N(R)=\sum_{k=1}^{R} 6 k+1=6 \frac{R(R+1)}{2}+1=3 R(R+1)+1
$$

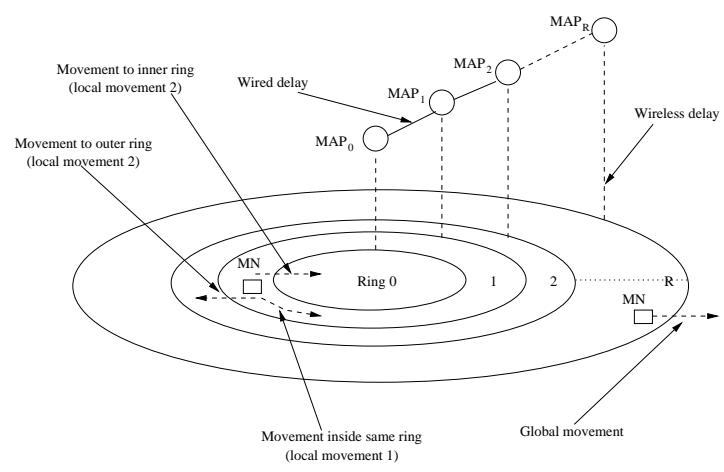

Fig. 4. An administrative domain with ring structure of access routers \& hierarchical structure of MAPs and the local \& global movements of an MN

We make another assumption that, there is one distinct MAP entity in each of the ( $\mathrm{R}+1)$ rings (including ring 0 ) which provides mobility management functions for the MNs roaming inside that particular domain. We further assume that, each MN inside a ring only has wireless link-delay while communicating with its current ring's MAP and each ring's MAP is connected by a wired link with its neighboring ring's MAP. As a result, an MN registered with an MAP other than its current ring's MAP, does not have only wireless link-delay part - it includes wired link-delay part also. So, the MAPs serving the MNs actually create a hierarchical structure. As we go higher in the hierarchy, the ring size increases (the number of access routers within each successive ring increases) and the coverage area within each successive ring, also becomes higher. Finally, we take all the wired link-delays within a domain to be same and wireless link-delay a bit larger than wired link-delay, since, the transmission cost in a wireless link is greater than that in a wired link.

\section{B. Random-walk Mobility Model}

Our analytical model is based on random-walk mobility model which is appropriate for pedestrian movements. According to this model, an MN moves to its adjacent cell with probability $p$ or remains in the current cell with probability 1-p. Based on the architecture of our domain, each movement decision of an $\mathrm{MN}$ results in any of the following 4 conditions:

- The MN stays in the same cell (i.e. the MN did not move)

- The MN stays in same ring (local movement 1 in Fig. 4)

- The MN moves to a cell of its neighboring ring (local movement 2 in Fig. 4)

- The MN moves out of the domain (global movement)
We assume that, the MN makes each movement decision only after its expected cell residence time. If an $\mathrm{MN}$ is located in a cell of ring $k(k>0)$, the probability that a movement of the MN will result in an increase, decrease or equality of distance from the center is given by

$$
\operatorname{Pr}^{+}(k)=\frac{2 k+1}{6 k}, \operatorname{Pr}^{-}(k)=\frac{2 k-1}{6 k} \& \operatorname{Pr}(k)=\frac{1}{3}
$$

We define state $k$ of a Markov chain as the index of a ring in which the $\mathrm{MN}$ is located. If we say, the $\mathrm{MN}$ is in state $k$, it implies that, the $\mathrm{MN}$ is currently located at any of the cells of ring $k$. Suppose, $\alpha_{k, k+1}$ and $\beta_{k, k-1}$ represent the probabilities at which the MN moves to the outer or inner neighboring ring respectively. Surely, $\alpha_{k, k+1}$ represents the joint probability of MN's movement and the increase of distance of the MN from the center. Similarly, $\beta_{k, k-1}$ represents the joint probability that the $\mathrm{MN}$ moves and its distance from the center decreases. Assume, $\gamma_{k, k}$ denotes the joint probability that the MN moves and its distance from the center remains the same.

$$
\begin{aligned}
& \alpha_{k, k+1}= \begin{cases}p & \text { if } k=0 \\
p\left(\frac{2 k+1}{6 k}\right) & \text { if } 1 \leq k \leq R\end{cases} \\
& \beta_{k, k-1}= \begin{cases}0 & \text { if } k=0 \\
p\left(\frac{2 k-1}{6 k}\right) & \text { if } 1 \leq k \leq R\end{cases} \\
& \gamma_{k, k}= \begin{cases}0 & \text { if } k=0 \\
p\left(\frac{1}{3}\right) & \text { if } 1 \leq k \leq R\end{cases}
\end{aligned}
$$

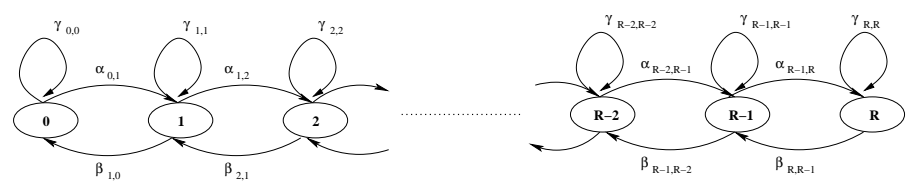

Fig. 5. State diagram for random walk mobility model

Figure 5 shows the states \& associated transition probabilities regarding our random-walk mobility model. Suppose, $P_{k}$ denotes the steady state probability of state $k$ among $(R+1)$ possible states of the Markov chain model. In other words, $P_{k}$ defines the steady-state probability of an MN to be inside ring $k$ of an administrative domain comprising $R$ rings. Using transition probabilities of Eq. 1 and Eq. $2, P_{k}$ can be expressed as,

$$
P_{k}=P_{0} \prod_{i=0}^{k-1} \frac{\alpha_{i, i+1}}{\beta_{i+1, i}} \text { for } 1 \leq k \leq R
$$

We know from Markov chain property that, the summation of all steady-state probabilities equals to 1 . With this requirement, $P_{k}$ can be obtained as, 


$$
P_{k}=\frac{\prod_{i=0}^{k-1} \frac{\alpha_{i, i+1}}{\beta_{i+1, i}}}{1+\sum_{k=1}^{R} \prod_{i=0}^{k-1} \frac{\alpha_{i, i+1}}{\beta_{i+1, i}}}
$$

\section{Cost Functions}

In this section, at first, we analyze the unit handover delay for one micro or macro movement and, thereby, obtain the cost functions (based on L3 handover delay) for our concerned protocols - MIPv6, HMIPv6, [F+H]MIPv6 and XMAP-HMIPv6. We assume that, $E(T)$ is the average cell residence time of an $\mathrm{MN}$ and, $\delta \& \tau$ denote unit transmission delay in a wired and wireless link respectively $(\tau>\delta)$. Here, the handover latency is assumed to be the "L3 handover delay" which is defined as the time, required for the $\mathrm{MN}$ to acquire new validated $\mathrm{CoA}$ to communicate with a $\mathrm{CH}$ at the IP level.

\section{A. Mobile IPv6}

In standard MIPv6 protocol, whenever the MN goes through a handover, the MN must get registered with the HA entity of its home network first. Then, return routability procedure is run between the $\mathrm{MN}$ and all of its $\mathrm{CHs}$, after which, the $\mathrm{MN}$ will eventually update its binding with the CHs. So, the handover latency of an MN for any movement in MIPv6 protocol,

$$
\begin{aligned}
D_{M I P v 6}= & 2 \times\left[D_{M N-H A}+D_{M N-C H}+\right. \\
& \left.M a x\left(D_{M N-C H}, D_{M N-H A}+D_{H A-C H}\right)\right]
\end{aligned}
$$

Here, $D_{M N-H A}, D_{M N-C H}$ and $D_{H A-C H}$ represent the linkdelay between $\mathrm{MN}$ and $\mathrm{HA}, \mathrm{MN}$ and $\mathrm{CH}$, and $\mathrm{HA}$ and $\mathrm{CH}$ respectively. In our analytical mobility model, each local or global movement of the MN causes same amount of delay. So, the cost equation takes the following form,

$$
\mathcal{C}_{M I P v 6}=\frac{p \times D_{M I P v 6}}{E(T)}
$$

Where $p$ and $E(T)$ denote the probability of the MN's movement and the MN's expected cell residence time respectively.

\section{B. Hierarchical Mobile IPv6}

In HMIPv6, an MN performs two types of binding update - global and local. For our analytical model's local movement $1 \& 2$ (i.e. micro mobility), the MN only needs to update its binding with its currently registered MAP. So, the handover latency for the MN's micro-movement equals the binding update procedure time with the MAP. For global binding update (i.e. macro mobility), the MN first registers with a local MAP and thereby, obtains a RCoA on the MAP's link. After that, the MN registers its RCoA with the HA and the $\mathrm{CHs}$. So, for macro-mobility, we get,

$$
D_{H M I P v 6-\text { global }}=2 \times[\tau+R \delta]+D_{M I P v 6}
$$

For our analytical model, we assume that, every time the MN moves into a new administrative domain providing HMIPv6 facilities, it registers with the Gateway MAP in the hierarchy (i.e. with the farthest MAP). According to the mobility model proposed, the probability that an $\mathrm{MN}$ performs the global binding update is $P_{R} \times \alpha_{R, R}$ and the probability that an MN performs local binding update is the summation of $P_{i} \times \alpha_{i, i+1}$, $P_{i+1} \times \beta_{i+1, i}$ (local movement 2) and $P_{i} \times \gamma_{i, i}$ (local movement 1). So, the cost equation becomes,

$$
\begin{aligned}
\mathcal{C}_{H M I P v 6}= & \frac{1}{E(T)}\left(\sum_{i=1}^{R}\left[\gamma_{i, i} \times P_{i} \times 2(\tau+(R-i) \delta)\right]+\right. \\
& \alpha_{R, R+1} \times P_{R} \times D_{H M I P v 6-\text { global }}+ \\
& \sum_{i=0}^{R-1}\left[\alpha_{i, i+1} \times P_{i} \times 2(\tau+(R-i-1) \delta)+\right. \\
& \left.\left.\beta_{i+1, i} \times P_{i+1} \times 2(\tau+(R-i) \delta)\right]\right)
\end{aligned}
$$

\section{Combination of Fast \& Hierarchical Mobile IPv6}

We consider the combination of predictive fast handover and HMIPv6. In case of micro mobility, the handover latency is only the wireless link-delay, $\tau$ (i.e. the delay to announce the MN's presence in the new subnet). In case of macro mobility, the delay is similar to the HMIPv6's macro mobility handover latency (i.e. $D_{H M I P v 6-g l o b a l}$ ). So,

$$
\begin{aligned}
\mathcal{C}_{[F+H] M I P v 6}= & \frac{1}{E(T)}\left(\left(p-\alpha_{R, R+1}\right) \times \tau+\right. \\
& \left.\alpha_{R, R+1} \times P_{R} \times D_{H M I P v 6-\text { global }}\right)
\end{aligned}
$$

\section{Cross-over MAP based Hierarchical Mobile IPv6}

Similar kind of analysis holds as HMIPv6 but only the delay components for the micro and macro-mobility are different. In case of micro-mobility, the binding update only has to travel to the cross-over MAP. Considering our mobility model, the latency component when the MN moves inside a ring or moves to its outer neighboring ring is $2 \tau$ but, when it moves to the inner neighboring ring, the component becomes $2(\tau+\delta)$. For macro-mobility, the latency component is equal to $D_{M I P v 6}$. So, the cost equation,

$$
\begin{aligned}
\mathcal{C}_{X M A P-H M I P v 6}= & \frac{1}{E(T)}\left(\sum_{i=1}^{R}\left[\gamma_{i, i} \times P_{i} \times 2 \tau\right]+\right. \\
& \alpha_{R, R+1} \times P_{R} \times D_{M I P v 6}+ \\
& \sum_{i=0}^{R-1}\left[\alpha_{i, i+1} \times P_{i} \times 2 \tau+\right. \\
& \left.\left.\beta_{i+1, i} \times P_{i+1} \times 2(\tau+\delta)\right]\right)
\end{aligned}
$$

\section{Numerical Results}

In this section, we present numerical results based on our random-walk mobility model which show the impacts of movement probability of $\mathrm{MN}$, its cell residence time, domain size and speed (i.e. both movement and cell residence time of $\mathrm{MN}$ ) on the handover latency costs. For the analysis, we consider some of the system parameters as constants (e.g. $D_{M N-H A}=10, D_{M N-C H}=20, D_{H A-C H}=5, \delta=1$, $\tau=2$ ). We have also assumed the movement probability, $p=0.5$, expected cell residence time, $E(T)=6 \mathrm{~ms}$ and the 
ring size, $R=7$ when they are not considered as the variable parameter.
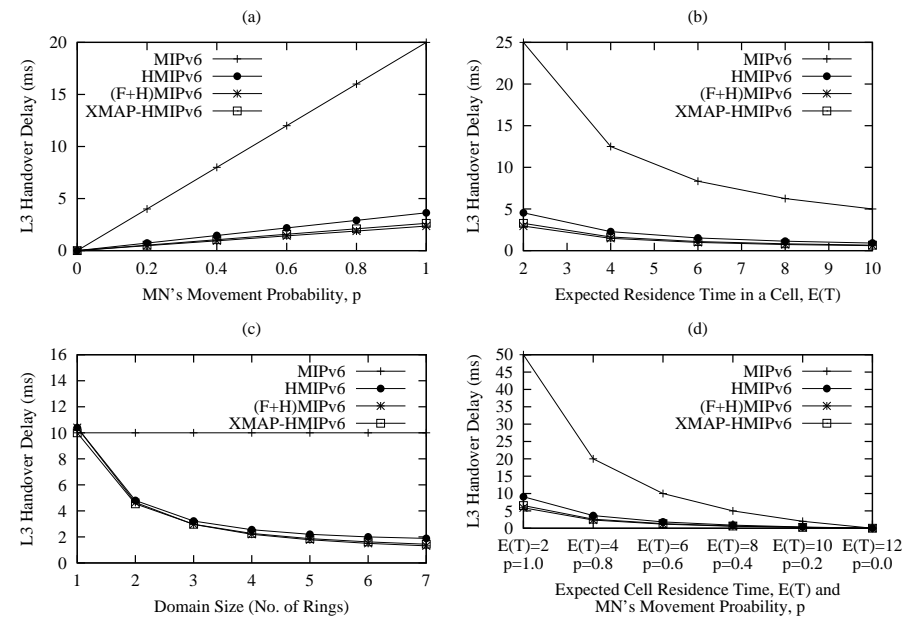

Fig. 6. Handover latency as a function of $p, E(T)$, domain size \& speed

\section{A. The Impact of MN's movement probability, $p$}

In Fig. 6(a), we see that, handover latency cost increases linearly with the MN's movement probability. We further see that, MIPv6 performs the worst among all the concerned protocols and our protocol, XMAP-HMIPv6 performs almost similar to the combination, $(\mathrm{F}+\mathrm{H}) \mathrm{MIPv} 6$.

\section{B. The Impact of Cell Residence Time, $E(T)$}

The average cell residence time is the period of time that an $\mathrm{MN}$ is expected to stay in a cell area. Thus, as the average cell residence time increases, the $\mathrm{MN}$ performs less movement and consequently, the handover latency cost per unit time decreases for all the protocols. In a comparative basis, we see that our protocol, XMAP-HMIPv6, performs close to $(\mathrm{F}+\mathrm{H}) \mathrm{MIPv} 6$ and outperforms both HMIPv6 and MIPv6.

\section{The Impact of Domain Size}

Apart from MIPv6, the handover latency cost for the other protocols are inversely proportional to the domain size as shown in Fig. 6(c). In case of MIPv6, the cost remains constant as MIPv6 does not differentiate a movement inside a domain from a movement across domains. We further see that, XMAPHMIPv6 performs better than HMIPv6 and closely matches with the combination, (F+H)MIPv6.

\section{The Impact of Speed of the MN}

A dynamic MN stays in the current cell with low probability and also, the MN's expected cell residence time is short. On the contrary, a static MN has high probability of staying at the current cell and the expected cell residence time is quite large. From Fig. 6(d), we find that, in case of dynamic MNs, the cost is significantly large while considering MIPv6. But as the MN tends to be static, all the costs become smaller and the difference of the costs for the concerned protocols also becomes less.

\section{CONCLUSION AND FUtURE WORK}

From the analytical model results, we find that, XMAPHMIPv6 performs almost similar to $[\mathrm{F}+\mathrm{H}] \mathrm{MIPv6}$. But, $[\mathrm{F}+\mathrm{H}] \mathrm{MIPv6}$ 's performance largely depends on the correct anticipation of the imminent handover. $[\mathrm{F}+\mathrm{H}] \mathrm{MIPv} 6$ also suffers from "synchronization" problem - when the MAP should stop forwarding to the PAR and start forwarding to NAR. Buffer management is also an issue for [F+H]MIPv6 where the NAR must buffer the packets destined for the MN if the MN does not get attached to it quickly enough. On the other hand, XMAP-HMIPv6 always finds the closest MAP on the path from $\mathrm{MN}$ towards HA to divert the traffic, thereby, resulting in small handover latency. It neither requires the correct anticipation of the handover nor suffers from the other mentioned problems that $[\mathrm{F}+\mathrm{H}] \mathrm{MIPv} 6$ tends to suffer.

Though the analytical results are proven satisfactory for our protocol, substantial work still remains to be done. One direction for further study can be to implement XMAPHMIPv6 and analyze the upper-layer protocols' (TCP, UDP etc.) performance using it. In our scheme, every packet destined for the MN is encapsulated by all the registered MAPs. If the number of registered MAPs is large, the packet size may become significant. The binding table lookup time for each MAP may also contribute to the delay of each arriving packet for the MN. So, another direction for further study can be to extend our analysis to "packet delivery cost" concerning all the protocols.

\section{REFERENCES}

[1] C. Perkins, "IP Mobility Support for IPv4", RFC 3344, August 2002.

[2] D. Johnson, C. Perkins and J. Arkko, "Mobility Support in IPv6", RFC 3775, June 2004.

[3] Hesham Soliman, Claude Castelluccia, Karim El-Malki and Ludovic Bellier, "Hierarchical Mobile IPv6 mobility management (HMIPv6)", draft-ietf-mobileip-hmipv6-07.txt (work in progress), October 2002.

[4] Miska Sulander, Timo Hamalainen, Ari Viinikainen and Jani Puttonen, "An Analysis of the Flow-based Fast Handover Method for Mobile IPv6 Network", in Proceedings of the 1st International Conference on Ebusiness and Telecommunication Networks, August 2004.

[5] Miska Sulander, Timo Hamalainen, Ari Viinikainen and Jani Puttonen, "Flow-based fast handover method for mobile IPv6 network", in Proceedings of the 59th IEEE Semiannual Vehicular Technology Conference (VTC'S04), May 2004.

[6] Postel J., 'Internet Protocol - DARPA Internet Program Protocol Specification", RFC 791, DARPA, September 1981.

[7] Rajeev Koodli, "Fast Handovers for Mobile IPv6", draft-ieft-mobileipfast-mipv6-08.txt (work in progress), October 2003.

[8] S. Deering and R. Hinden, "Internet Protocol Version 6 (IPv6) Specification", RFC 2460, December 1998.

[9] S. Thomson and T. Narten, "IPv6 Stateless Address Autoconfiguration", RFC 2462, December 1998.

[10] Sangheon Pack and Yanghee Choi, "Performance Analysis of Hierarchical Mobile IPv6 in IP-based Cellular Networks", in Proc. IP-Based Cellular Networks (IPCN) Conference 2003, Paris, France, December 2003.

[11] Sheldon M. Ross, "Introduction to Probability Models", Amsterdam, Academic Press, 2003. 